Running Head: IMPROVING RELATIONAL INTEGRATION

Does solving distant analogies facilitate relational integration?

\author{
Glenda Andrews* \& Gal Rachel Bohadana \\ School of Applied Psychology, Griffith University, Gold Coast, Australia \\ $\&$
}

Menzies Health Institute Queensland, Griffith University, Gold Coast, Australia

* Corresponding author

School of Applied Psychology, Griffith University, Gold Coast, 4222, Australia

Tel: +61-7-56788613, e-mail: g.andrews@griffith.edu.au 


\begin{abstract}
Relational processing underpins many forms of human thinking. This research addressed whether relational integration in the $n$-term task (linear syllogisms with three and four premises) can be facilitated. We hypothesized that solving distant analogies (e.g., furnace : coal :: stomach :___ but not near analogies (e.g., furnace : coal :: woodstove :__ would facilitate $n$-term performance of undergraduates $(N=120)$. Participants generated solutions to near analogies (near condition), to distant analogies (distant condition) while participants in the control condition completed a word rating task. Participants then completed the $n$-term task with items at two levels of complexity (ternary, quaternary), and a fluid intelligence test. Solving distant analogies facilitated relational integration on the more complex quaternary-relational items. It eliminated the complexity effect in the $n$-term task and the association between quaternary-relational reasoning and fluid intelligence.
\end{abstract}

Keywords: relational integration, analogy, semantic distance, relational reasoning; linear syllogisms 


\section{Does solving distant analogies facilitate relational integration?}

Relational processing underpins many forms of human thinking, problem solving and processes such as categorization, planning, language, and some executive functions (Halford, Wilson, \& Philips, 2010). It involves recognizing meaningful relations contained in streams of information and applying or integrating those relations (Alexander, Dumas, Grossnickle, List, \& Firetto, 2016; Holyoak, 2012). One example is the series completion task. This task requires individuals to recognize the relations among a series of digits such as 12834656 _ _ and apply the identified relations (ascending by one and descending by two) to continue the series (e.g., 4, 7). Relational reasoning is also used when solving analogies. An example of an analogy problem is ash: fireplace::lint: ?. To solve the problem, the reasoner first identifies the relation between ash and fireplace and then maps the identified relation to generate a potential solution (e.g., pocket).

Research examining whether and how relational reasoning might be facilitated is limited. Given the demonstrated involvement of relational reasoning in a range of content domains, including those that are relevant to training in the science, technology, engineering and mathematics (STEM) disciplines (Dumas, Alexander, \& Grossnickle, 2013), the benefits to society of promoting relational reasoning would be considerable. The current research addressed the issue of whether relational integration can be facilitated.

Formally, a relation is a binding between a relation symbol and a set of related elements (Goodwin \& Johnson-Laird, 2005; Halford, Wilson, \& Phillips, 1998; 2010). For example, the larger_than relation entails a binding between the symbol, larger_than and the set of ordered pairs; $\{.$. (elephant, mouse), ... (mountain, mole-hill) ... $\}$. Relational processing has properties that distinguish it from more basic processes such as association (Halford et al., 1998; Halford, Wilson, Andrews \& Phillips, 2014). Two such properties that are relevant to the current research are its effortful nature and its domain-general quality. 
The effortful nature of relational processing stems from its complexity. The complexity of a relation depends on the number of arguments. Unary relations such as class membership, fish(salmon), have a single argument. Binary relations consist of two arguments as in larger-than(lion, mouse). Ternary relations integrate three arguments as in arithmetic addition(4,5,9). Quaternary relations involve four arguments as in proportion(2,3,6,9). Relational complexity theory holds that as complexity increases, so too does the processing load, therefore relational processing becomes more effortful (Halford et al., 1998; 2014). The capacity to process more complex relations increases during childhood (Bunch \& Andrews, 2012; Richland, Morrison \& Holyoak, 2006), declines in later adulthood (Todd, 2011; Viskontis, Holyoak \& Knowlton, 2005), and it is sensitive to frontal lobe damage (Andrews, Halford, Shum, Maujean, Chappell, \& Birney, 2013; Waltz, Knowlton, Holyoak, Boone, Mishkin, de Menezes Santos et al., 1999). Quaternary-relational processing appears to be the upper limit for most adults, although some individuals process more complex quinaryrelations under optimal conditions (Halford, Baker, McCredden \& Bain, 2005).

Relational complexity effects have been demonstrated in many different content domains including transitive inference (Andrews \& Halford, 2002; Viskontis,et al., 2005; Waltz, et al., 1999), suppositional reasoning (Birney \& Halford, 2002), reasoning in a Latin Square task (Birney, Halford, \& Andrews, 2006), categorical syllogisms (Zielinski, Goodwin, \& Halford, 2010), conditional reasoning (Coochi et al., 2014; Cutmore et al., 2015), balance scale reasoning (Andrews, Halford, Murphy, \& Knox, 2009; Halford, Andrews, Dalton, Boag, \& Zielinski, 2002), numerical reasoning (English \& Halford, 1995), analogical reasoning (Richland, Morrison \& Holyoak, 2006), problem solving in the Tower of London task (Andrews, Halford, Chappell, Maujean, \& Shum, 2014) and sentence comprehension (Andrews, Birney \& Halford, 2006; Andrews, Ogden \& Halford, 2017). 
There is limited research concerning whether and how relational reasoning might be facilitated. A recent study (Vendetti, Wu \& Holyoak, 2014) suggests that short term facilitation is possible. Vendetti et al. examined the impact of analogical thinking on subsequent relational reasoning in a picture mapping task. In the picture mapping task, pairs of scenes were presented simultaneously. One object in the upper scene was highlighted and participants were asked to choose an object in the lower scene that "goes with" the highlighted object. Participants could make either relational or featural responses. Relational matches differed from the highlighted objects but they served the same role. For example, an umbrella is one scene and a newspaper in the other each sheltered a person from the rain. Featural matches were identical to the highlighted objects, but they served different roles in the two scenes.

Analogy problems were presented prior to the picture mapping task. In Experiment 1a, either near analogies (e.g., bracelet:wrist :: ring:finger) or distant analogies (e.g., bracelet:wrist :: moat:castle) were presented and participants evaluated the analogies as valid or invalid. There was no significant difference in relational choices on the picture mapping task between the near and distant analogy conditions in Experiment 1a. In Experiment 1b, the first three terms $\left(\mathrm{A}: \mathrm{B}:: \mathrm{C}: \_\right)$were presented but the final $(\mathrm{D})$ term in each analogy was omitted. Participants in the near and distant conditions generated solutions to the incomplete analogies. There were significantly more relational choices on the picture mapping task in the distant analogy condition than in the near analogy condition in Experiment 1b (Vendetti et al., 2014).

Vendetti et al. (2014) concluded that generating solutions to distant analogies was an effective way to "induce a relational mindset” that influenced performance on a different relational reasoning task that used unrelated materials. They proposed that solving near analogies involves matching identical relations in the source $(\mathrm{A}: \mathrm{B})$ and target $(\mathrm{C}$ : _ $)$ to 
generate the D term. For example, the same relation “worn on” can be applied to the source (bracelet:wrist) and target (ring:__ in the near analogy because bracelet and ring are both jewelry items. Matching identical relations is not sufficient for distant analogies (bracelet:wrist :: moat: ) which require relations that are more abstract or more general to span the greater semantic distance between source and target structures. The higher rate of relational responses in the picture mapping task appears to stem from the experience gained by generating these more abstract relations in the distant analogy condition of Experiment $1 \mathrm{~b}$.

A further finding was that for participants in the near analogy condition, the proportion of relational responses made on the picture mapping task was predicted by fluid intelligence. Similar associations between relational processing and fluid intelligence have been observed in other research (Andrews, 2010; Andrews \& Halford, 2002; Birney, Beckmann, Beckmann \& Double, 2017; Birney, Bowman, Beckmann \& Seah, 2012; Hansell et al., 2015). Such findings support the view that performance on tests of relational reasoning and fluid intelligence reflect a stable underlying capacity. However, this was not the case in the distant analogy condition, where the association between fluid intelligence and relational responding on the picture mapping task was not significant. Generating solutions to distant analogies eliminated the association between fluid intelligence and relational choices on the picture mapping task. Vendetti et al's (2014) interpretation was that this was due to the induction of a relational mindset, which implies a short-term rather than long-lasting change. Nevertheless the resulting increase in relational responding was sufficient to break the association with fluid intelligence in the distant analogy condition.

Current understanding of analogy and its component processes is informed by a large body of behavioural and computational research in cognitive science (Gentner, 1983; Gick \& Holyoak, 1980; Holyoak, 2012; Sternberg, 1977) and more recently by research in cognitive neuroscience (e.g., Green, 2016; Green, Kraemer, Fugelsang, Gray \& Dunbar, 2010, 2012; 
Whitaker, Vendetti, Wendelken \& Bunge, 2017). Three processes that have emerged as important in analogy are controlled semantic retrieval, inhibitory control, and relational integration (Whitaker et al., 2017). These three processes have been associated with activation in different sub-regions of the prefrontal cortex. The relative importance of these processes varies according to procedural and task parameters (e.g., evaluation of analogies versus generation of solutions; presence of perceptual distractors) as well as participant characteristics such as information processing capacities and their familiarity with the relations in the analogies (Goswami \& Brown, 1989).

Research by Green and colleagues has demonstrated the importance of relational integration in distant analogies, which they described as a form of creative reasoning. Relational integration is needed to combine the relation between the $\mathrm{A}$ and $\mathrm{B}$ terms with the relation between the $\mathrm{C}$ and $\mathrm{D}$ terms to form a single abstract representation of their similarity (Green, 2016). In the example above, the relation between bracelet and wrist would be integrated with the relation between moat and castle to form an abstract relation such as "surrounds". Relational integration would be less important in the near analogies where identical relations can be matched (Vendetti et al., 2014) without the need to identify a more general or abstract relation. In two studies, Green and colleagues presented analogies that varied in semantic distance. Participants evaluated the analogies (Green et al., 2010) or generated solutions to them (Green et al., 2012) while undergoing fMRI scanning. In both studies, greater semantic distance was associated with increased activity in the left frontopolar cortex. These effects of semantic distance were dissociable from those of difficulty. In earlier research the same frontopolar regions had been implicated in relational integration in a range of reasoning tasks (e.g., Bunge, Wendelken, Badre \& Wagner, 2005; Christoff, et al., 2001; Kroger, Sabb, Fales, Bookheimer, Cohen \& Holyoak, 2002). 
Other research (Green, Cohen, Kim \& Gray, 2012) demonstrated that providing explicit cues to "be creative” facilitated performance on distant analogies. This facilitation was interpreted as a change in state creative reasoning. This dynamic creative state was distinguished from the more static trait of creativity. To the extent that distant analogies entail relational integration, this finding suggests that short-term changes in relational integration can be achieved.

The purpose of the current study was to examine whether generating solutions to distant analogies facilitates relational integration. As noted, distant analogies are seen as more creative than near analogies by virtue of the greater semantic distance that they span (Green, Cohen et al., 2012) and fMRI studies (Green et al., 2010, 2012) support the interpretation that distant analogies impose higher relational integration demands than near analogies. Vendetti et al. (2014) concluded that solving the distant analogies induced a relational mind-set that influenced a different relational reasoning task, but they did not make the more specific claim that relational integration was facilitated. The picture mapping task appears to require mapping of similar or identical relations. As such, it would not impose a high relational integration demand.

In the current research, we used a relational reasoning task that focuses more directly on relational integration. The $n$-term premise integration task (Andrews et al., 2006; Hansell et al., 2015) is a linear syllogistic reasoning task. Premises (e.g., T $<$ P, P $>$ V, T $<$ V) are presented and participants are required to integrate the premises and construct an ordered sequence of the elements (e.g., $\mathrm{P}>\mathrm{V}>\mathrm{T}$ ). Each premise relates two elements that are either immediately adjacent $(\mathrm{P}>\mathrm{V} ; \mathrm{T}<\mathrm{V})$ in the sequence or are nonadjacent $(\mathrm{T}<\mathrm{P})$. The nonadjacent premise spans more positions in the sequence (greater distance) than the adjacent premises. The $n$-term task resembles the distant analogies in that both require integration of relations over distance, spatial distance in the former and semantic distance in the latter. $\mathrm{N}$ - 
term items at two levels of relational complexity were presented. The example given above is a ternary-relational item in which an ordered sequence of three elements is constructed. In quaternary-relational items, there are four premises, and ordered sequences of four elements are constructed.

The $n$-term task is similar to transitive inference in some respects. Constructing an ordered sequence that integrates the premises is a key component of transitive inference (Maybery, Bain \& Halford, 1986). In the $n$-term task this is also the response elicited from participants, but transitive inference tasks usually require a different type of response. In standard transitive inference tasks, the premises (e. g., P $>$ V, T $<$ V) would be presented then participants would be asked to infer the relation between a pair of elements (e.g., Which is larger, $\mathrm{P}$ or T?).

Prior to the $n$-term task, participants completed one of three tasks. Participants in the control condition completed a word rating task. Participants in the near analogy condition generated solutions to near analogies and those in the distant condition generated solutions to distant analogies. Fluid intelligence was also assessed.

If generating solutions to distant analogies facilitates relational integration, then accuracy on the $n$-term task will be higher in the distant analogy condition, compared to the near analogy condition and the control condition. If solving analogies (near or distant) facilitates relational integration, then accuracy on the $n$-term task will be higher in the near and distant analogy conditions compared to the control condition. If facilitation is observed, two further questions will be examined. First, does the facilitation effect differ across the levels of complexity (ternary, quaternary) of the $n$-term items? Second, is the facilitation effect sufficiently strong to eliminate the association between accuracy on the $n$-term task and fluid intelligence in the distant analogy condition?

\section{Method}




\section{Participants}

The initial sample consisted of 123 individuals. The data of three individuals were excluded. One participant's responses to the quarternary $n$-term problems were not recorded, due to a technical failure. Two individuals gave no correct responses on the $n$-term task. The final sample consisted of 120 students (41 males, 79 females) enrolled in a first-year psychology course at Griffith University, who received course credit for their participation. The mean age in years was $22.12(S D=5.95)$, and $88 \%$ of participants reported having English as their first language. There were 40 participants in each of the control, near analogy, and distant analogy conditions. Chi-square tests revealed there were no significant associations between gender and condition, $\chi^{2}(2, N=120)=0.07, p=.96$, nor between first language and condition, $\chi^{2}(2, N=120)=0.65, p=.72$. A one-way ANOVA revealed that the conditions did not differ significantly in terms of the participants' age, $F(2,117)=0.27, p=$ .70 .

\section{Materials \& Procedure}

The research was approved by the Griffith University Human Research Ethics Committee (GU ref no: 2016/300). Participants were tested in groups of up to five, and each group was randomly assigned to either the control condition, the near analogy condition, or the distant analogy condition. The duration of each session was approximately 50 minutes.

Participants in each condition were allocated a personal computer, and were then asked to read the information sheet and to indicate their consent by signing the form. They provided demographic information regarding their age, gender, and first language. The next step differed according to condition.

Participants in the control condition rated the pleasantness of trait adjectives sourced from Anderson (1968). Participants were presented with a list of 63 randomly ordered adjectives that described positive (i.e., generous) and negative (i.e., boastful) traits. 
Participants were asked to rate each adjective in terms of its pleasantness on a scale from 1 to 7, where 1 represented very unpleasant and 7 represented very pleasant. The first two items were presented for practice, and once participants understood the task requirements they rated the remaining adjectives.

The materials used in the near and distant analogy conditions were drawn from Green, et al., (2010). The current study utilized 40 near analogy problems and 40 distant analogy problems. Each analogy was presented in the format of A:B::C: where letters represented words and “___ is, furnace:coal::woodstove: . An example of a distant analogy is furnace:coal::stomach: . Participants in the analogy conditions were asked to generate solutions to the analogies by providing a suitable D term for each problem (e.g., "wood” for the near analogy and "food" for the distant analogy). Analogies were presented in booklets and participants provided written responses. Two practice items with feedback after each were presented in each analogy condition, to ensure understanding of the task requirements. The remaining 38 problems were the test problems. In both the near and distant analogy conditions, participants were allowed 15 minutes to complete as many analogies as possible. Responses (D terms) were considered correct if they matched the solutions provided Green et al. (2010) or if the D terms formed a good analogy when combined with the A, B, and C, terms. The maximum score possible was 38 .

Participants in all conditions then completed the relational integration task, followed by the fluid intelligence test.

$N$-term. Relational integration was assessed using the $n$-term premise integration task (Andrews et al., 2006; Hansell et al., 2015). On each trial, three or four premises were presented on the left side of the screen. Each premise consisted of two alphabetic letters related by either a < or $>$ sign. Participants were required to construct descending sequences 
of three or four alphabetic letters. The order of the letters in the constructed sequence had to be consistent with the premise information. Examples of 3-term and 4-term items are shown in Figure 1. The 3-term and 4-term items have been analyzed as requiring ternary-relational and quaternary-relational processing, respectively. The task procedures were designed to minimize opportunities for reducing complexity using chunking and segmentation strategies (Andrews et al., 2006). Participants read the premises at their own pace then entered their responses. Instructions emphasized accuracy. Participants were instructed to construct the entire sequence mentally before commencing their responses because responses could not be changed. The premises remained in view until the final key stroke of the response.

Participants were not permitted to make notes as they solved the problems. One practice item and eight test items at each complexity level were presented. The dependent measures were solution accuracy $(\max =8)$ and median solution times for correct solutions.

Insert Figure 1 about here

Fluid intelligence was assessed using the Culture Fair Test, Scale 2, Form A (Cattell \& Cattell, 1973). The stimuli in the four subtests were drawings of geometric objects. In each subtest, either two or three practice items with feedback were presented prior to the test items. The Series subtest had 12 test items. On each item, an incomplete series of three stimuli was presented and participants chose one of five options that best continued the series. The Classifications subtest had 14 test items. On each item, five figures were presented and participants selected the one that was different from the other four. The Matrices subtest had 12 test items. On each item, an incomplete $2 \times 2$ matrix was presented 
and participants chose one of five options to complete the matrix. The Conditions subtest had 8 test items. On each item, participants selected from the five choices provided, the one that duplicated the conditions (spatial position of a dot in relation to various shapes) given in the box on the far left. The subtests were administered using the standard instructions and the time limits specified in the manual. Participants were instructed to complete as many test items as possible within the allocated times which were 3 minutes (Series), 4 minutes (Classifications), 3 minutes (Matrices) and 2.5 minutes (Conditions). The test required induction of the relations between the stimuli and inferences based on these relations. Correct responses on the four subtests were summed to yield a total score $(\max =46)$.

\section{Results}

\section{Preliminary Analyses}

To check whether participants in the control condition complied with task instructions for the word-rating task, one-sample $t$-tests were conducted to compare the ratings given to negative and positive words to the neutral midpoint of 4 . The $t$-tests confirmed the positive words were rated as significantly more pleasant $(M=5.66, S D=0.49), t(39)=18.40, p<$ .001 , and the negative words as significantly less pleasant $(M=2.32, S D=0.47), t(39)=-$ 19.81, $p<.001$ than the scale midpoint.

A one-way ANOVA confirmed that significantly more correct solutions were generated to the near analogies $(M=26.18, S D=8.64)$ than to the distant analogies $(M=$ 18.50, $S D=6.74), F(1,78)=19.62, p<.001, \eta^{2}=.20$.

A one-way ANOVA revealed that participants in the three conditions did not differ significantly in terms of fluid intelligence, $F(2,117)=0.41, p=.67$. Descriptive statistics are provided in Table 1.

\section{Main Analyses}


$N$-term accuracy. Correct solutions were analyzed using a 3 (Condition) $\times 2$ (Complexity) mixed factorial ANOVA with condition as the between-groups factor and complexity as the within-subjects factor. There were significant main effects of complexity, $F(1,117)=31.63, p<.001, \eta_{p}^{2}=.21$, and condition, $F(2,117)=4.27, p=.016, \eta_{p}^{2}=.07$. These main effects were modified by a significant Complexity $\times$ Condition interaction, $F(2$, $117)=3.33, p=.039, \eta^{2}=.05$. The means are shown in Table 1.

Insert Table 1 about here

The interaction was examined further. The main effect of condition was significant for the more complex quaternary-relational items, $F(2,117)=5.72, p=.004, \eta^{2}=.09$, but not for the less complex ternary-relational items, $F(2,117)=1.51, p=.226$. Follow-up $t$-tests revealed that accuracy on the quaternary-relational items was higher in the distant analogy condition than the control condition $t(39)=3.36, p=.001, d=.73$ and the near analogy condition, $t(39)=1.99, p=.048, d=.49$. There was no significant difference in accuracy between the control and near analogy conditions, $t(39)=1.37, p=.174$.

Complexity effects were significant in the control condition, $F(1,117)=22.27, p<$ $.001, \eta^{2}=.16$ and the near analogy condition, $F(1,117)=14.56, p<.001, \eta^{2}=.11$. In these two conditions accuracy was lower for quaternary-relational than for ternary-relational items. However the complexity effect was not significant in the distant analogy condition, $F(1,117)=1.45, p=.231$.

$N$-term solution times. Median solution times were calculated on the ternaryrelational and quaternary-relational items. These were based on correct responses only, therefore two participants who made no correct responses on quaternary-relational items were 
not included in this analysis. A mixed 3 (Condition) $\times 2$ (Complexity) ANOVA with median solution times as the dependent variable yielded a significant main effect of complexity, $F(1$, $115)=169.59, p<.001, \eta_{p}^{2}=.60$, but no significant main effect of condition, $F(2,115)=$ $0.97, p=.38$, and no significant interaction between condition and complexity, $F(2,115)=$ $0.39 p=.68$. Median solution times (seconds) were significantly longer for the quaternaryrelational $(M=33.90, S E=13.10)$ than the ternary-relational items $(M=18.22, S E=7.46)$. Descriptive statistics for the three conditions are provided in Table 1.

Associations with fluid intelligence. Zero-order correlations between $n$-term accuracy and fluid intelligence were computed. In the full sample $(N=120)$ fluid intelligence was positively associated with accuracy on quaternary-relational $(r=.33, p<.001)$ and ternary-relational $n$-term items $(r=.22, p=.018)$. In the control condition, fluid intelligence was significantly correlated with accuracy on the quaternary-relational $n$-term items $(r=.31$, $p=.05)$ and the correlation with ternary-relational $n$-term items was not significant $(r=.20, p$ $=.22)$. In the near analogy condition, fluid intelligence was significantly correlated with accuracy on the quaternary-relational $n$-term items $(r=.49, p=.001)$ and the correlation with ternary-relational $n$-term items approached significance $(r=.29, p=.067)$. In the distant analogy condition, fluid intelligence was not significantly correlated with accuracy on the quaternary-relational $n$-term items $(r=.17, p=.29)$ nor the ternary-relational $n$-term items $(r$ $=.13, p=.42)$.

\section{Discussion}

The current study investigated whether relational integration could be facilitated by generating solutions to analogies. There were three main findings. The first was that generating solutions to distant analogies facilitated relational integration in the $n$-term task. Relative to the near analogy and the control conditions, participants in the distant analogy condition had greater success when integrating premise information to construct ordered 
sequences. By contrast, generating solutions to near analogies conferred no benefit relative to the control condition. This indicates that the facilitation stemmed from the greater semantic distance that the distant analogies spanned. Our favored interpretation is that solving distant (not near) analogies provided experience in integrating relations across semantic distance and that this experience facilitated relational integration across spatial distance in the $n$-term task.

The second finding is that the facilitation in the distant analogy condition was observed primarily on the more complex quaternary-relational items. The relational integration demand on these items was higher because there were more premises to integrate. This is consistent with previous research based on Relational Complexity theory showing that quaternary-relational processing is more difficult than ternary-relational processing and that the quaternary-relational level constitutes the upper limit for most young adults (Halford, et al., 2005). The higher accuracy on the quaternary-relational items meant that the complexity effect was eliminated in the distant analogy condition. Thus brief experience solving distant analogies was sufficient to overcome the complexity effect that is usually observed on this task (Andrews, 2010; Andrews et al., 2006; Hansell et al., 2015) and which was significant in the near analogy and control conditions in the current experiment.

The finding of higher $n$-term accuracy after solving distant (but not near) analogies is consistent with Vendetti et al's (2014) finding of increased relational responding on the picture mapping task. However, as noted previously the relational integration demands of the picture mapping and $n$-term tasks appear to differ. In the picture mapping task, similar or identical relations are mapped. In the example described above, the relation (shelter) in the upper scene (umbrella shelters woman from rain) also applies in the lower scene (newspaper shelters woman from rain) and there is no need to form a more abstract or more general relation. By contrast, the premises of the $n$-term task relate both adjacent and nonadjacent elements in the constructed sequences. To generate each sequence, the relations spanning 
shorter and longer distances must be recognized and integrated. Relational integration in the $n$-term task resembles that required in distant analogies in which relations that span greater semantic distances are needed (Green, 2016)

Our claim that relational integration in distant analogies is better captured by the $n$ term task than by the picture mapping task should not be seen as dismissing Vendetti et al.’s (2014) important findings or their interpretation that the distant analogies induced a relational mindset that facilitated relational responding on the picture mapping task. Rather, it seems plausible that experience on a task that requires more difficult relational integration (distant analogies) would facilitate relational responding on a task that requires less difficult relational integration (picture mapping) to a greater extent than a task that requires less difficult relational integration (near analogies) would.

Our findings for the $n$-term task are also consistent with Greene, Cohen et al.’s (2012) finding that explicit cues to be creative resulted in more correct solutions to distant analogies. As argued above both tasks require relational integration across distances. The facilitation observed by Green et al was interpreted as a change in state rather than trait. The short duration of the change is consistent with Vendetti et al's interpretation that solving distant analogies induced a relational mindset. The change observed in the current study is likely to be similarly short in duration. Further research using more complex designs and larger samples is required to determine the precise duration of the effect.

An alternative interpretation is that the observed difference in $n$-term accuracy might be due to carry-over of the effort expended on the first task. Solving distant analogies might require more effort than solving near analogies, which might require more effort than rating the adjectives. While this interpretation seems plausible, there are some arguments against it. Solutions times provide a potential indicator of effort on the $n$-term task, but they did not differ significantly between the three conditions. The expected direction of the effect of effort 
is unclear. To the extent that participants expended greater effort on the first task they might have become fatigued and consequently expended less (not more) effort on subsequent tasks. Finally, according to the effort interpretation, $n$-term accuracy should be higher in the near analogy condition than in the control condition, but this was not the case.

Nevertheless, further investigation of the effort explanation is warranted. Our primary reason for including the control condition was to assess whether solving near analogies would confer any benefit on $n$-term performance relative to a non-analogy condition - an issue not addressed by Vendetti et al. (2014). The adjective rating task is comparable to the near analogy task in that the stimuli were verbal and the pleasantness ratings requires semantic processing. It remains possible that the word rating task required less effort than the near analogies task, although as already noted this did not impact on $n$-term performance. Our word rating condition was not intended as a control for effort expended solving distant analogies. Future research could examine the effort explanation more directly using a different control task that is comparable to distant analogies in terms of effort. Indicators of the effort expended during the initial tasks and the relational integration task could also be included

The third finding is that the experience of solving distant analogies was sufficient to eliminate the association between fluid intelligence and $n$-term performance on quaternaryrelational items. The significant associations with $n$-term performance in the control and near analogy conditions of the current research likely reflects stable individual differences in relational reasoning. Similar associations with this and other relational reasoning tasks have been observed in previous research (Andrews, 2010; Andrews \& Halford, 2002; Birney et al., 2012, 2017). In the distant analogy condition, the corresponding association was not significant. One explanation is that the temporary facilitation due to generating solutions to distant analogies was uneven, affecting some individuals more so than others. Those with 
higher fluid intelligence would likely have performed well even without the experience of solving distant analogies, so there was little scope for these individuals to perform better on the $n$-term task. In contrast, individuals with lower fluid intelligence would have performed less well if they had not solved the distant analogies, so there was more scope for their performance to benefit from doing so. Their $n$-term scores would resemble those of individuals with higher fluid intelligence, thereby eliminating the association between the two tasks which would otherwise be observed. This explanation could be examined further using a pre- and post-test design in which individuals with higher and lower fluid intelligence attempt the $n$-term task before and after solving the analogies.

Vendetti et al. (Experiment 1b, 2014) also found that the association with fluid intelligence was eliminated in the distant analogy condition but it remained significant in the near analogy condition. Thus in both studies, the short-term facilitation in relational reasoning produced by generating solutions to distant analogies was sufficient to eliminate the association with fluid intelligence.

The facilitation in relational reasoning observed in the current study has potential implications in non-laboratory contexts. For example, many university students struggle to understand and interpret statistical interactions. Halford et al. (2005) argued that the difficulty stems from the complex relations that interactions entail. Having students solve distant analogies just prior to tackling this material might assist students to acquire an initial understanding, which could be consolidated and elaborated further through practice with examples. The distant analogies could be used as warm up exercises prior to teaching 2-way interactions and again when more complex 3-way interactions are introduced. After many repetitions in this and other contexts, the temporary relational mind set or state induced by distant analogies might become a more stable and enduring disposition to think in terms of relations. 
Our experiment extends previous research in several ways. It demonstrates that facilitation of relational reasoning as a result of solving distant analogies is not restricted to the picture mapping task used by Vendetti et al. (2014), but also occurs on the $n$-term test. The picture mapping and $n$-term tasks differ in various ways including their stimuli (pictures versus alphabetic letters and symbols), response types (choose an object versus construct a sequence) and procedural details. Furthermore we have proposed that the tasks differ in terms of their relational integration demands. However, because each task involves relational processing, we expected a similar effect. Given the domain-general nature of relational reasoning facilitation would be expected on a wide range of relational tasks.

There is also scope to examine modes of relational reasoning other than analogies. Alexander and colleagues (Alexander et al., 2016; Dumas et al., 2013) treat relational reasoning as a multidimensional construct. They distinguished analogies (based on similarity relations) from anomalies (based on discrepancy relations), antinomies (based on incompatibility relations), and antithesis (based on opposition). Future research could examine the extent to which experience with these separate, but related modes of relational reasoning enhance relational integration in the $n$-term task

Our research also demonstrates the value of including items at different levels of complexity. This increases the sensitivity of measures of relational integration and increases the likelihood that facilitation will not go undetected due to ceiling or floor effects that might result in tasks whose complexity is either too high or too low. Future research could examine whether the facilitation effect observed here for quaternary-relational items in young adults extends to more complex quinary-relational items or whether there is an upper limit on facilitation. There is also scope for further examination of facilitation of ternary-relational processing. Whereas young adults (on average) operate at or near the quaternary-relational level (Halford et al., 2005), this is not the case for young children (e.g., Bunch \& Andrews, 
2012) older adults (e.g., Todd, 2011; Viskontis et al., 2005) and people with frontal lobe lesions (e.g., Andrews et al., 2013; 2014; Waltz et al., 1999; 2004) who experience difficulty with ternary-relational tasks. Future research could investigate whether facilitation effects extend to the ternary level of complexity in these populations. Generation of analogy solutions may be especially beneficial for older people. Analogies rely heavily on semantic knowledge (Holyoak \& Koh, 1987), which is relatively immune to age-related decline (Roskos, Ewoldsen, Black, \& McCown, 2008).

More generally, the research demonstrated the value of employing facilitation techniques that are informed by theory and knowledge of the underlying cognitive processes when investigating the extent to which relational processing can be improved (Andrews, Halford \& Boyce, 2012; Andrews \& Moussaumai, 2015). The domain-general quality of relational reasoning and findings from the current research indicate that experience in one content domain can facilitate performance in a different content domains. To the extent that the facilitation observed here provides the foundation for longer term improvements in relational reasoning, there would be widespread benefits for improving educational outcomes, particularly those relevant to STEM disciplines.

\section{Disclosure statement}

No potential conflict of interest was reported by the authors 


\section{References}

Alexander, P. A., Dumas, D., Grossnickle, E. M., List, A. \& Firetto, C. (2016). Measuring relational reasoning. The Journal of Experimental Education, 84, 119-151.

Anderson, N. (1968). Likeableness ratings for 555 personality trait words. Journal of Personality and Social Psychology, 9, 272-279.

Andrews, G. (2010). Belief-based and analytic processing in transitive inference depends on premise integration difficulty. Memory \& Cognition, 38, 928-940.

Andrews, G., Birney, D., \& Halford, G. S. (2006). Relational processing and working memory capacity in comprehension of relative clause sentences. Memory and Cognition, 34, 1325-1340.

Andrews, G., Halford, G.S., (2002). A cognitive complexity metric applied to cognitive development. Cognitive Psychology, 45, 153-219.

Andrews, G., Halford, G. S., \& Boyce, J. (2012). Conditional discrimination in young children: The roles of associative and relational processing. Journal of Experimental Child Psychology, 112, 84-101.

Andrews, G., Halford, G. S., Chappell, M., Maujean, A., \& Shum, D. H. (2014). Planning following stroke: A relational complexity approach using the Tower of London. Frontiers in Human Neuroscience, 8. 1-14.

Andrews, G., Halford G. S., Murphy, K., \& Knox, K. (2009) Integration of weight and distance information in young children: The role of relational complexity. Cognitive Development, 24, 49-60.

Andrews, G., Halford, G. S., Shum, D., Maujean, A., Chappell, M., \& Birney, D. (2013). Relational processing following stroke. Brain and Cognition, 81, 44-51. 
Andrews, G., \& Moussaumai, J. (2015). Improving children’s affective decision making in the Children’s Gambling Task. Journal of Experimental Child Psychology, 139, 1834. doi:10.1016/j.jecp.2015.05.005

Andrews, G., Ogden, J. E. \& Halford, G. S. (2017) Resolving conflicts between syntax and plausibility in sentence comprehension. Advances in Cognitive Psychology, 13, 11-27.

Birney, D. P., \& Halford, G. S., (2002). Cognitive complexity of suppositional reasoning: An application of the relational complexity metric to the knight-knave task. Thinking and Reasoning, 8, 109-134.

Birney, D. P., Halford, G. S., \& Andrews G. (2006). Measuring the influence of complexity on relational reasoning: The development of the Latin Square Task. Educational and Psychological Measurement, 66, 146-171.

Birney, D. P., Beckmann, J. F., Beckmann, N., \& Double, K. S. (in press, 2017). Beyond the intellect: Complexity and learning trajectories in Raven’s Progressive Matrices depend on self-regulatory processes and conative dispositions. Intelligence. http://dx.doi.org/10.1016/jintell.2017.01.005

Birney, D. P., Bowman, D. B., Beckmann, J., \& Seah, Y. (2012). Assessment of processing capacity: Latin-square task performance in a population of managers. European Journal of Psychological Assessment, 28, 216-226

Bunch, K. M., \& Andrews, G. (2012). Development of relational processing in hot and cool tasks. Developmental Neuropsychology, 37, 134-152.

Bunge, S. A., Wendelken, C., Badre, D., \& Wagner, A. D. (2005). Analogical reasoning and prefrontal cortex: Evidence for separable retrieval and integration mechanisms. Cerebral Cortex, 15, 239-249.

Cattell, R. B., \& Cattell, A. K. S. (1973). Measuring intelligence with the culture fair tests. Institute for Personality and Ability Testing. Champaign, Illinois. 
Christoff, K., Prabhakaran V., Dorfman, J., Zhao Z., Kroger, J. K., Holyoak, K. J., Gabrieli, J.D. (2001). Rostrolateral prefrontal cortex involvement in relational integration during reasoning. Neuroimage 14, 1136--1149.

Coochi, L. et al., (2014). Complexity in relational processing predicts changes in functional brain network dynamics. Cerebral Cortex, 24, 2283-2296.

Cutmore, T. R. H., Halford, G. S., ...Spokes, T., \& Shum, D. H. K. (2015). Neural correlates of deductive reasoning: An ERP study with the Wason Selection Task. International Journal of Psychophysiology, 98, 381-388.

Dumas, D., Alexander, P. A., \& Grossnickle, E. M. (2013). Relational reasoning and its manifestations in the educational context: A systematic review of the literature. Educational Psychology Review, 25, 391-427.

English L. D., \& Halford, G. S. (1995). Mathematics education: Models and processes. Hillsdale, NJ: Erlbaum.

Gentner, D. (1983). Structure-mapping: A theoretical framework for analogy. Cognitive Science, 7, 155-170.

Goodwin, G. P., \& Johnson-Laird, P. N. (2005). Reasoning about relations. Psychological Review, 112, 468-493.

Goswami, U., \& Brown, A. L. (1989). Melting chocolate and melting snowmen: Analogical reasoning and causal relations. Cognition, 35, 69-95.

Green, A. E. (2016). Creativity, within reason: Semantic distance and dynamic state creativity in relational thinking and reasoning. Psychological Science, 25, 28-35.

Green, A. E., Cohen, M. S., Kim, J. U., \& Gray, J. R. (2012). An explicit cue improves creative analogical reasoning. Intelligence, 40, 598-603. 
Green, A. E., Kraemer, D. J., Fugelsang, J. A., Gray, J. R., \& Dunbar, K. N. (2010). Connecting long distance: semantic distance in analogical reasoning modulates frontopolar cortex activity. Cerebral Cortex, 20, 70-76.

Green, A. E., Kraemer, D. J., Fugelsang, J. A., Gray, J. R., \& Dunbar, K. N. (2012). Neural correlates of creativity in analogical reasoning. Journal of Experimental Psychology: Larning, Memory \& Cognition, 38, 264-272.

Halford G. S., Andrews, G., Dalton, C., Boag, C., \& Zielinski, T. (2002) Young children’s performance on the balance scale: The influence of relational complexity. Journal of Experimental Child Psychology, 81, 417-445.

Halford, G. S., Baker, R., McCredden, J. E. \& Bain, J. D. (2005). How many variables can humans process? Psychological Science, 16, 70-76.

Halford, G. S., Wilson, W. H., Andrews, G., \& Phillips, S. (2014). Categorizing cognition: Toward conceptual coherence in the foundations of psychology. Cambridge, MA: The MIT Press.

Halford, G. S., Wilson, W. H., \& Phillips, S. (1998). Processing capacity defined by relational complexity: Implications for comparative, developmental, and cognitive psychology. Behavioral and Brain Sciences, 21(6), 803-831.

Halford, G. S., Wilson, W. H., \& Phillips, S. (2010). Relational knowledge: The foundation of higher cognition. Trends in Cognitive Sciences, 14, 497-505.

Hansell, N. K., Halford, G. S., Andrews, G., Shum, D. H., Harris, S. E., Davies, G., ... \& Medland, S. E. (2015). Genetic basis of a cognitive complexity metric. PloS One, 10. Holyoak, K. J. (2012). Analogy and relational reasoning. In K.J. Holyoak \& R.G. Morrison (Eds.), The Oxford Handbook of Thinking and Reasoning (pp. 234-259). New York, NY: Oxford University Press. 
Holyoak, K. J., \& Koh, K. (1987). Surface and structural similarity in analogical transfer. Memory \& Cognition, 15, 332-340. doi:10.3758/BF03197035

Kroger, J. K., Sabb, F. W., Fales, C. I., Bookheimer, S. Y., Cohen, M. S., \& Holyoak, K. J. (2002). Recruitment of anterior dorsolateral prefrontal cortex in human reasoning: a parametric study of relational complexity. Cerebral Cortex, 12, 477- 485.

Maybery, M. T., Bain, J. D. \& Halford, G. S. (1986). Information processing demands of transitive inference. Journal of Experimental Psychology: Learning , Memory \& Cognition, 12, 600-613.

Richland, L E., Morrison, R. G., \& Holyoak, K. J. (2006). Children’s development of analogical reasoning: Insights from scene analogy problems. Journal of Experimental Child Psychology, 94, 249-273.

Roskos-Ewoldsen, B., Black, S. R., \& McCown, S. M. (2008). Age-related changes in ceative thinking. The Journal of Creative Behavior, 42, 33-59. doi:10.1002/j.21626057.2008.tb01079.x

Sternberg, R. J., (1977). Intelligence, Information Processing, and Analogical Reasoning. New York, NY: Plume.

Todd, J. A. (2011). Age Related Changes During Adulthood in Cognitive Processes Reliant on the Pre-Frontal Cortex: Attention, Inhibitory Control, Working Memory and Relational processing. (Unpublished doctoral dissertation). Griffith University, Gold Coast.

Vendetti, M. S., Wu, A., \& Holyoak, K. J. (2014). Far-out thinking: Generating solutions to distant analogies promotes relational thinking. Psychological Science, 25, 928-933.

Viskontis, I. V., Holyoak, K. J., \& Knowlton, B. J. (2005). Relational integration in older adults. Thinking \& Reasoning, 11, 390-410. 
Waltz, J. A., Knowlton, B. J., Holyoak, K. J., Boone, K. B., Back-Madruga, C., McPherson, S., ... \& Miller, B. L. (2004). Relational integration and executive function in Alzheimer's disease. Neuropsychology, 18, 296-305. doi:10.1037/0894-4105.18.2.296

Waltz, J. A., Knowlton, B. J., Holyoak, K. J., Boone, K. B., Mishkin, F. S., de Menezes Santos, M., ... \& Miller, B. L. (1999). A system for relational reasoning in human prefrontal cortex. Psychological science, 10, 119-125. doi:10.1111/1467-9280.00118

Whitaker, K. J., Vendetti, M. S., Wendelken, C., \& Bunge, S. (2017). Neuroscientific insights into the development of analogical reasoning. Developmental Science, doi:10.1111/desc 12531

Zielinski, T. A., Goodwin, G. P., \& Halford G. S., (2010). Complexity of categorical syllogisms: An integration of two metrics. European Journal of Cognitive Psychology, 22, 391-421. 
Table 1.

Descriptive statistics (means and standard errors) for accuracy (maximum $=8$ ) and median solution times (secs) on ternary- and quaternary-relational n-term items and fluid intelligence $($ maximum $=46)$

\section{Condition}

Control Near analogy $\quad$ Distant analogy

$N$-term

Ternary-relational accuracy

$6.58(.25)$

$6.95(.25)$

$7.18(.25)$

Quaternary-relational accuracy

$5.40(.31)$

$6.00(.31)$

$6.88(.31)$

Ternary-relational solution times

$19.10(1.30)$

$18.93(1.30)$

$16.64(1.28)$

Quaternary-relational solution times

$36.00(2.28)$

$33.22(2.28)$

$32.47(2.25)$

Fluid intelligence

$35.33(0.71)$

$35.23(0.71)$

36.05 (0.71) 


\section{Figure Captions}

Figure 1. Example 3-term and 4-term items in the $n$-term premise integration task. 


\begin{tabular}{ccc}
\hline Premises & Correct sequence & Complexity \\
\hline $\mathbf{T}<\mathbf{P}$ & $\mathbf{P}>\mathbf{V}>\mathbf{T}$ & 3-term \\
$\mathbf{P}>\mathbf{V}$ & & (ternary) \\
$\mathbf{T}<\mathbf{V}$ & $\mathbf{F}>\mathbf{B}>\mathbf{A}>\mathbf{X}$ & 4-term \\
$\mathbf{B}>\mathbf{A}$ & & (quaternary) \\
$\mathbf{A}<\mathbf{F}$ & & \\
$\mathbf{F}>\mathbf{B}$ & \\
$\mathbf{X}<\mathbf{A}$ &
\end{tabular}

